\section{EL CÁNCER DE MAMA}

\author{
Miguel Martín \\ Hospital General Universitario Gregorio Marañón \\ mmartin@geicam.org \\ Ana Herrero \\ Hospital General Universitario Gregorio Marañón \\ anahh16@hotmail.com \\ Isabel Echavarría \\ Hospital General Universitario Gregorio Marañón \\ iechavarriadg@gmail.com
}

Cómo citar este artículo/Citation: Martín, M.; Herrero, A.; Echavarría, I. (2015). "El cáncer de mama". Arbor, 191 (773): a234. doi: http://dx.doi.org/10.3989/arbor.2015.773n3004

Recibido: 9 diciembre 2014. Aceptado: 24 abril 2015.

RESUMEN: El cáncer de mama es un problema socio-sanitario de primer orden en España debido a su elevada incidencia. En las últimas tres décadas se han producido notables mejoras en la supervivencia debidas tanto a la introducción de las campañas de cribado mamográfico como a los nuevos tratamientos médicos. Pese a estos datos optimistas, no hay que olvidar que aún quedan pacientes que recaen tras el tratamiento del tumor mamario (cerca del $20 \%$ ) y fallecen a causa de la enfermedad, por lo que es importante continuar con los esfuerzos de investigación en cáncer de mama hasta conseguir una tasa de recaída cero. En este artículo se revisan distintos aspectos de esta enfermedad como son sus síntomas y signos, el diagnóstico, la quimioprevención, así como el tratamiento del cáncer de mama localizado y metastásico.

PALABRAS CLAVE: carcinoma ductal; carcinoma lobulillar; HER2; tratamiento.

\section{BREAST CANCER}

Copyright: (C) 2015 CSIC. Este es un artículo de acceso abierto distribuido bajo los términos de la licencia Creative Commons Attribution-Non Commercial (by-nc) Spain 3.0.
ABSTRACT: The high incidence of breast cancer makes it a major social-health problem in Spain. Over the last three decades, survival rates have improved markedly thanks to the introduction of mammographic screening campaigns and new treatments. Despite the optimistic data, we should not forget that there some patients still relapse after their breast cancer treatment (about 20\%), some of whom die from the disease. It is therefore important to continue breast cancer research efforts until a relapse rate of zero is achieved. In this article, we review various aspects of this disease such as its signs and symptoms, diagnosis, chemoprevention, as well as the treatment of localised and metastatic breast cancer.

KEYWORDS: ductal carcinoma; HER2; lobular carcinoma; treatment. 


\section{INTRODUCCIÓN}

Por su elevada incidencia, el cáncer de mama es un problema socio-sanitario de primer orden en España. En las últimas tres décadas se han producido notables mejoras en la supervivencia debidas tanto a la introducción de las campañas de cribado mamográfico, como a los nuevos tratamientos médicos. Ello es particularmente relevante teniendo en cuenta que la incidencia de cáncer de mama ha aumentado en nuestro país en este periodo. Pese a estos datos optimistas, no hay que olvidar que aún quedan pacientes que recaen tras el tratamiento del tumor mamario (cerca del $20 \%$ ) y fallecen a causa de la enfermedad, por lo que es importante continuar con los esfuerzos de investigación en esta enfermedad hasta conseguir una tasa de recaída cero.

El cáncer de mama no es una enfermedad, sino una familia de enfermedades con al menos 4 tipos moleculares bien definidos (Perou et al., 2000), los tumores hormonodependientes (o luminales, que se subclasifican a su vez en luminales $A$ y B), los tumores con amplificación del oncogén HER2 y los tumores denominados triple negativos. El 65\% de los tumores de mama tienen fenotipo luminal; es decir, son HER2 negativos con receptor hormonal positivo, el $18-20 \%$ tiene sobre-expresión del receptor HER2 y el $15 \%$ restante son tumores del tipo triple negativo (HER2 negativo con receptor hormonal negativo). Estos receptores se evalúan de forma rutinaria en todos los informes anatomopatológicos de cáncer de mama y son fundamentales para predecir el riesgo, y determinar la estrategia de tratamiento.

\section{EPIDEMIOLOGÍA DEL CÁNCER DE MAMA}

El cáncer de mama es el tumor maligno más frecuente en las mujeres, con más de 1,2 millones de casos diagnosticados cada año en el mundo. Este cáncer produce unas 500.000 muertes anuales en todo el mundo, siendo la primera o segunda causa de muerte por cáncer en mujeres dependiendo de los países (está por detrás del carcinoma de pulmón en muchos países desarrollados) (Ferlay et al., 2007). Globalmente, el cáncer de mama es el tumor más frecuente entre las mujeres de todo el mundo, (22,7\% del total de cánceres femeninos) según datos de la Organización Mundial de la Salud. Si consideramos los dos sexos, solo le superan los cánceres de pulmón, estómago y cánceres colorrectales. La supervivencia a 5 años en nuestro país está próxima al $85 \%$.

En Europa, la probabilidad de una mujer de desarrollar un cáncer de mama antes de los 75 años es del
8-10\%. Su incidencia aumenta con el nivel económico, de tal forma que más de la mitad de los casos de cáncer de mama se diagnostican en los países desarrollados: 370.000 casos al año en Europa $(27,4 \%)$ y 230.000 en Norteamérica (31,3\%). La incidencia en España es de las más bajas, aunque ha aumentado progresivamente en las últimas décadas. En España, se calculan unos 25.000 nuevos casos anuales (lo que supone una cuarta parte de todos los casos de cáncer femenino) y unas 5.000 muertes anuales debidos al tumor. La mayoría de los casos se diagnostican entre los 35 y los 80 años de edad, con un máximo entre los 45 y los 65 . En nuestro país, no existe un patrón geográfico claro, destacando solamente la provincia de Gran Canaria como área de mayor mortalidad por cáncer de mama.

Existen unos factores de riesgo de padecer cáncer de mama, lo que permite identificar a las mujeres con mayor predisposición que puedan entrar en programas de vigilancia que permitan su diagnóstico precoz, o incluso valorar cirugías de reducción de riesgo (en el caso de las mujeres que pertenecen a familias con cáncer hereditario de mama/ovario).

- Edad: la incidencia aumenta de forma progresiva con la edad hasta los 45-50 años, en que tiende a estabilizarse. En España, la edad media de las mujeres en el momento del diagnóstico es de 56 años. Las campañas de cribado mamográfico comienzan por ello a los 50 años en la mayoría de las comunidades y se extienden hasta los 65 o 70 años.

- Nivel socioeconómico y lugar de residencia: las mujeres con más alto nivel educacional, ocupacional y económico, así como las que viven en áreas urbanas presentan mayor riesgo de padecer cáncer de mama.

- Patrón de paridad "occidental": embarazos poco numerosos, el primero en edades muy tardías. En España se ha detectado un aumento de la incidencia de cáncer de mama en mujeres menores de 45 años (18,6\% del total de las mujeres diagnosticadas de cáncer de mama entre 1998 y 2001) (Martin et al., 2004), a diferencia de otros países europeos donde esta incidencia se ha estabilizado. La posible explicación a este cambio producido en España lo encontramos en las modificaciones en el estilo de vida de las mujeres españolas con unas tasas de natalidad muy bajas, con una media de 1,2 embarazos (Bosch, 1998; Pollan et al., 
2009), siendo además el país europeo donde más se retrasa la edad del primer embarazo (Arroyo, 2003; Hery et al., 2008; Levi et al., 2007; Soerjomataram et al., 2008).

- Estilo de vida: se ha asociado un mayor riesgo de cáncer de mama en mujeres con sobrepeso y vida sedentaria.

- Factores hereditarios: la existencia de familiares con cáncer de mama y/o ovario (especialmente familiares de primer grado) es un factor de riesgo importante que aumenta el riesgo de padecer cáncer de mama. Cuanto mayor sea el grado de relación y más temprana la aparición de casos en una familia, mayor probabilidad habrá de que otro miembro de la familia padezca cáncer de mama. Un tercio de las pacientes con historia familiar de cáncer de mama y/o ovario son portadoras de una mutación heredable en los genes BRCA1 o BRCA2. En las mujeres sanas pertenecientes a familias con mutaciones BRCA1-2 y que tengan también la mutación, el riesgo a largo plazo de tener cáncer de mama es muy alto (superior al 60\%), por lo que deben someterse a un seguimiento específico en Unidades de Consejo Genético Oncológico. Las mutaciones de cualquiera de estos genes también aumentan el riesgo de contraer cáncer del ovario. Existen Unidades de Consejo Genético donde se planifica la información y el seguimiento de las personas pertenecientes a estas familias, a la vez que se determina si se deben realizar pruebas genéticas para detectar las mutaciones. Las recomendaciones para el manejo de estas mujeres es diferente al de la población general (por ejemplo, la opción de cirugía reductora del riesgo, bien mastectomía bilateral profiláctica, bien ovariectomía bilateral profiláctica o bien ambas debe ser discutida con las mujeres sanas portadoras de mutación y el seguimiento debe ser más estrecho).

- Lesiones mamarias indicadoras de riesgo: aumentan el riesgo de padecer cáncer de mama (adenosis esclerosante, papilomas intraductales, hiperplasia ductal atípica, hiperplasia lobulillar atípica y carcinoma lobulillar in situ).

- Tratamiento hormonal: La exposición prolongada a altas concentraciones de estrógenos eleva el riesgo de padecer cáncer de mama. Esto ocurre por ejemplo en mujeres con menarquia precoz, menopausia tardía y que no tienen hi- jos. No se han relacionado claramente los anticonceptivos o los programas de estimulación ovárica en tratamientos de fertilidad con un mayor riesgo de cáncer de mama. Sí que hay un aumento del riesgo relacionado con la terapia hormonal sustitutiva en la menopausia (aunque su cuantía depende del tipo de hormonas usadas y de la duración del tratamiento).

\section{HISTORIA NATURAL DEL CÁNCER DE MAMA}

El cáncer de mama se inicia usualmente en los conductos mamarios (y por ello se denomina carcinoma ductal), aunque un $10 \%$ se inicia en los lóbulos mamarios (y se denomina entonces carcinoma lobulillar). Se sospecha que la mayoría de los carcinomas ductales se inician en una forma no invasiva (y por tanto, no grave en sí misma) que se denomina carcinoma intraductal. Este carcinoma intraductal (que debería cambiar su nombre para evitar alarmas innecesarias) no puede extenderse a distancia dando metástasis y por tanto se cura siempre por resección local. Sin embargo, si no se trata, algunos carcinomas intraductales pueden transformarse con el tiempo en cáncer invasivo, originando un verdadero cáncer de mama. El cáncer invasivo o infiltrante de mama puede extenderse localmente dentro de la mama, puede llegar a infiltrar la piel o los músculos pectorales y puede también extenderse por los conductos linfáticos a los ganglios de la axila (mas raramente a los de la cadena ganglionar mamaria interna). Finalmente, el cáncer de mama puede invadir los vasos sanguíneos locales y emitir células metastásicas que pueden emigrar a órganos distantes (hueso, pleura, pulmón, hígado) dando lugar a metástasis a distancia.

De acuerdo con los niveles de extensión, la UICC clasifica el cáncer de mama en 4 estadios:

- Estadio I: tumores pequeños, sin afectación metastática de la axila.

- $\quad$ Estadio II: tumores de más de $2 \mathrm{~cm}$ o con afectación metastática de la axila moderada.

- Estadio III: tumores muy grandes o con afectación de piel o músculo pectoral o afectación axilar masiva.

- Estadio IV: metástasis en órganos distantes (hueso, pulmón, hígado...).

El pronóstico de estos estadios es muy diferente, con supervivencias aproximadas a 5 años de $95 \%$ (estadio I), $80 \%$ (estadio II), $60 \%$ (estadio III) y $25 \%$ (estadio IV). 


\section{SÍNTOMAS Y SIGNOS DEL CÁNCER DE MAMA}

El cáncer de mama no da síntomas o signos hasta que la enfermedad ha adquirido un notable desarrollo. Cuando aparecen, los signos más frecuentes de la enfermedad son:

(i) tumoración en la mama de reciente aparición (aunque hay que resaltar que la mayoría de las tumoraciones de mama que se descubre la mujer antes de la menopausia son benignas), (ii) cambios persistentes en la piel de la mama (enrojecimiento, retracción de la piel, piel de naranja),(iii) eccema del pezón, (iv) bultos en la axila, (v) hinchazón del brazo y (vi) retracción del pezón, secreciones anómalas por el pezón.

Si aparecen estos signos, la mujer debe acudir inmediatamente a su médico, aunque muchas veces se tratará de una falsa alarma, en particular en el caso de los bultos. Si se descubra en el periodo sintomático, el cáncer de mama puede aún ser curado, aunque en general precisará más tratamientos que si se descubre mediante una mamografía en periodo asintomático. Lo ideal es que el cáncer de mama se descubra antes de que produzca signos o síntomas y esto puede lograrse mediante las mamografías rutinarias de cribado (y en casos seleccionados, mediante otras pruebas (como la resonancia magnética nuclear en el caso de las mujeres pertenecientes a familias con cáncer de mama/ovario hereditarios). Cuando el cáncer se descubre en las campañas de cribado, las posibilidades de curación son muy altas (>90\%).

\section{DIAGNÓSTICO DE CONFIRMACIÓN}

La sospecha de la existencia de un cáncer de mama en las pruebas de imagen (mamografía, ecografía, resonancia magnética nuclear) obliga a realizar una confirmación histológica. Una biopsia con aguja gruesa es posible en la mayoría de los casos y permite conocer el tipo molecular de cáncer antes de decidir la hoja de ruta terapéutica de la enferma. En muy pocas ocasiones es necesario realizar el diagnóstico en el quirófano (biopsia quirúrgica) por imposibilidad de obtener una biopsia con aguja gruesa con control mediante técnicas de imagen. Mucho menos recomendable que la biopsia con aguja gruesa es la realización de una punción aspiración con aguja fina; está permite conocer la naturaleza de la lesión que se ha puncionado, sin embargo no proporciona información molecular.

Hay diferentes tipos histológicos de cáncer de mama. El tipo más frecuente de cáncer de mama es el carcinoma ductal (70-80\%) seguido del lobulillar (5-10\%) y otros menos frecuentes (medular, papilar, mucinoso y otros). Existe una forma pre-invasiva de patología mamaria denominada carcinoma "in situ" o carcinoma intraductal que no es un verdadero cáncer invasivo (si es una forma pura, nunca origina metástasis). No obstante, debe ser tratada mediante cirugía (y quizás también radioterapia), porque tiene el potencial de transformarse en un tumor invasivo.

Una vez realizada la confirmación mediante biopsia, debe procederse a determinar el estudio de extensión clínica del tumor, que permite tener una idea aproximada de la afectación de la mama y los ganglios linfáticos de drenaje. En los casos más avanzados o en caso de síntomas, está indicada la realización de pruebas encaminadas a detectar metástasis (TAC, ecografía, gammagrafía ósea). La determinación del estudio de extensión del cáncer de mama se realiza utilizando el sistema TNM de la AJCC (American Joint Comimittee on Cancer). $\mathrm{T}$ se refiere al tumor, $\mathrm{N}$ se refiere a los ganglios linfáticos y $\mathrm{M}$ se refiere a la presencia de metástasis en órganos distantes.

\section{CRIBADO DEL CÁNCER DE MAMA}

Las campañas de cribado poblacional de cáncer de mama se realizan invitando a todas las mujeres del censo que cumplen una cierta edad (50 años en la mayoría de los casos) a realizarse una mamografía bilateral de forma periódica (usualmente cada 2 años) hasta que cumplen los 70 años. Las campañas de cribado permiten el diagnóstico precoz de muchos cánceres, y aumentan la supervivencia entre un 25 y un $30 \%$ en las mujeres de edad igual o superior a 50 años. El beneficio de estas campañas antes de los 50 años y después de los 70 es más dudoso. La participación en estas campañas de cribado se sitúa en torno al $70 \%$ de la población invitada. Recientemente se ha puesto en entredicho la capacidad de las campañas de cribado para reducir la mortalidad por cáncer de mama y se han resaltado sus potenciales efectos indeseables, en particular la realización de biopsias innecesarias y el descubrimiento de cánceres poco agresivos que nunca darían problemas médicos a las mujeres si no fueran descubiertos pero que obligan a realizar un tratamiento oncológico clásico ya que son indistinguibles de los cánceres potencialmente peligrosos para la mujer. El menor impacto en supervivencia podría deberse a la eficacia de los nuevos tratamientos médicos, que logra que tumores avanzado que antes no se curaban ahora lo hagan. En la actualidad, se acepta que los beneficios del cribado compensan estos inconvenientes. Además del aumento de supervivencia, el cribado presenta la ventaja adicional de un diagnós- 
tico más precoz, lo que se traduce en una reducción de la agresividad del tratamiento oncológico.

\section{QUIMIOPREVENCIÓN DEL CÁNCER DE MAMA}

Existen muchas evidencias que implican a los estrógenos en la génesis del cáncer de mama. Por ello, varios estudios analizaron la capacidad de los fármacos antiestrogénicos orales para reducir la incidencia de cáncer de mama en mujeres sanas de alto riesgo para padecer la enfermedad. Tanto el tamoxifeno como el raloxifeno y el exemestano son capaces de reducir la incidencia de cáncer de mama. El tamoxifeno ha sido ensayado tanto en mujeres premenopáusicas como postmenopáusicas (Fisher et al., 1998), mientras que el raloxifeno y el exemestano solo han sido utilizados en mujeres postmenopáusicas. No se ha podido demostrar una reducción de la mortalidad por cáncer de mama asociada a la quimioprevención, aunque no puede descartarse. Los fármacos útiles en quimioprofilaxis no carecen de efectos secundarios, por lo que su uso debe analizarse individualmente. Se calcula que el tamoxifeno y raloxifeno evitan la aparición de un cáncer de mama aproximadamente por cada 80120 mujeres tratadas. El exemestano tiene menos efectos secundarios que el tamoxifeno y evita la aparición de un cáncer de mama por cada 96 mujeres tratadas (Goss et al., 2011).

\section{TRATAMIENTO DEL CÁNCER DE MAMA}

El tratamiento de los estadios I, II y III incluye dos aspectos: tratamiento local (cirugía +/- radioterapia) y tratamiento complementario o adyuvante, un tratamiento con medicamentos cuya composición depende de las características moleculares del tumor. El orden de estos tratamientos puede variar dependiendo del tamaño del tumor y del tipo molecular de cáncer y debe ser decidido de forma consensuada por un comité de expertos (Comité de Tumores) en el que haya al menos un cirujano o ginecólogo expertos en cáncer de mama, un oncólogo médico, un oncólogo radioterápico, un cirujano plástico e, idealmente, un patólogo y un radiólogo. Hoy en día, se intenta que ninguna mujer con cáncer de mama sea tratada sin que su caso haya sido previamente analizado por dicho Comité, y la mejor secuencia de tratamiento consensuada y registrada en las actas del Comité.

Los objetivos del tratamiento son producir la mayor tasa de curaciones de la enfermedad causando la menor cantidad de efectos adversos. Por ello, debe intentarse siempre la cirugía conservadora de la mama y la biopsia selectiva del ganglio centinela, dejando la amputación de la mama (mastectomía) y el vaciamiento axilar (linfadenectomía axilar) para casos muy seleccionados.

En los tumores luminales (los que expresan receptores de hormonas pero no HER2), el orden de terapias recomendable suele ser el siguiente:

1. En tumores candidatos a cirugía conservadora de la mama de inicio (tumores pequeños con una relación tamaño de la mama/tamaño del tumor favorable): tumorectomía (cirugía conservadora de la mama) y biopsia del ganglio centinela. La tumorectomía en la mayoría de los casos consigue un excelente resultado estético (Figura 1). La biopsia del ganglio centinela permite saber si hay afectación axilar por el tumor. Si es negativa, puede evitarse el vaciamiento axilar, que puede producir importantes secuelas de por vida. Tras ello, debe decidirse si la paciente debe recibir o no quimioterapia (los modernos tests genómicos, como Oncotype, Mammaprint, Prosigna y Endopredict son de gran ayuda para ello). Se administre o no quimioterapia, todas las enfermas deben recibir radioterapia sobre la mama conservada y tratamiento hormonal con pastillas durante al menos 5 años.

2. En tumores demasiado grandes para poder realizar cirugía conservadora de la mama, puede comenzarse el tratamiento con quimioterapia (denominada neoadyuvante) si la enferma desea conservarla. En el $70 \%$ de los casos, se produce una reducción del tumor y en el $40-50 \%$ puede procederse a la cirugía conservadora de la mama. Tras ello, las enfermas deben recibir radioterapia y tratamiento hormonal oral durante al menos 5 años.

En los tumores HER2-positivos, el orden de terapias suele ser:

1. En tumores candidatos a cirugía conservadora de la mama de inicio (tumores pequeños con una relación tamaño de la mama/tamaño del tumor favorable): tumorectomía (cirugía conservadora de la mama) y biopsia del ganglio centinela. Tras ello, la paciente debe recibir quimioterapia más terapia antiHER2 (trastuzumab) y radioterapia (también hormonas orales si el tumor además de HER2 expresa receptores de estrógenos).

2. En estos mismos tumores, aunque sean candidatos a cirugía conservadora de la mama de 
Figura 1. Tumorectomía del cuadrante superoexterno de la mama izquierda por un cáncer de mama de $2 \mathrm{~cm}$ (cirugía conservadora de la mama). La enferma recibió después radioterapia

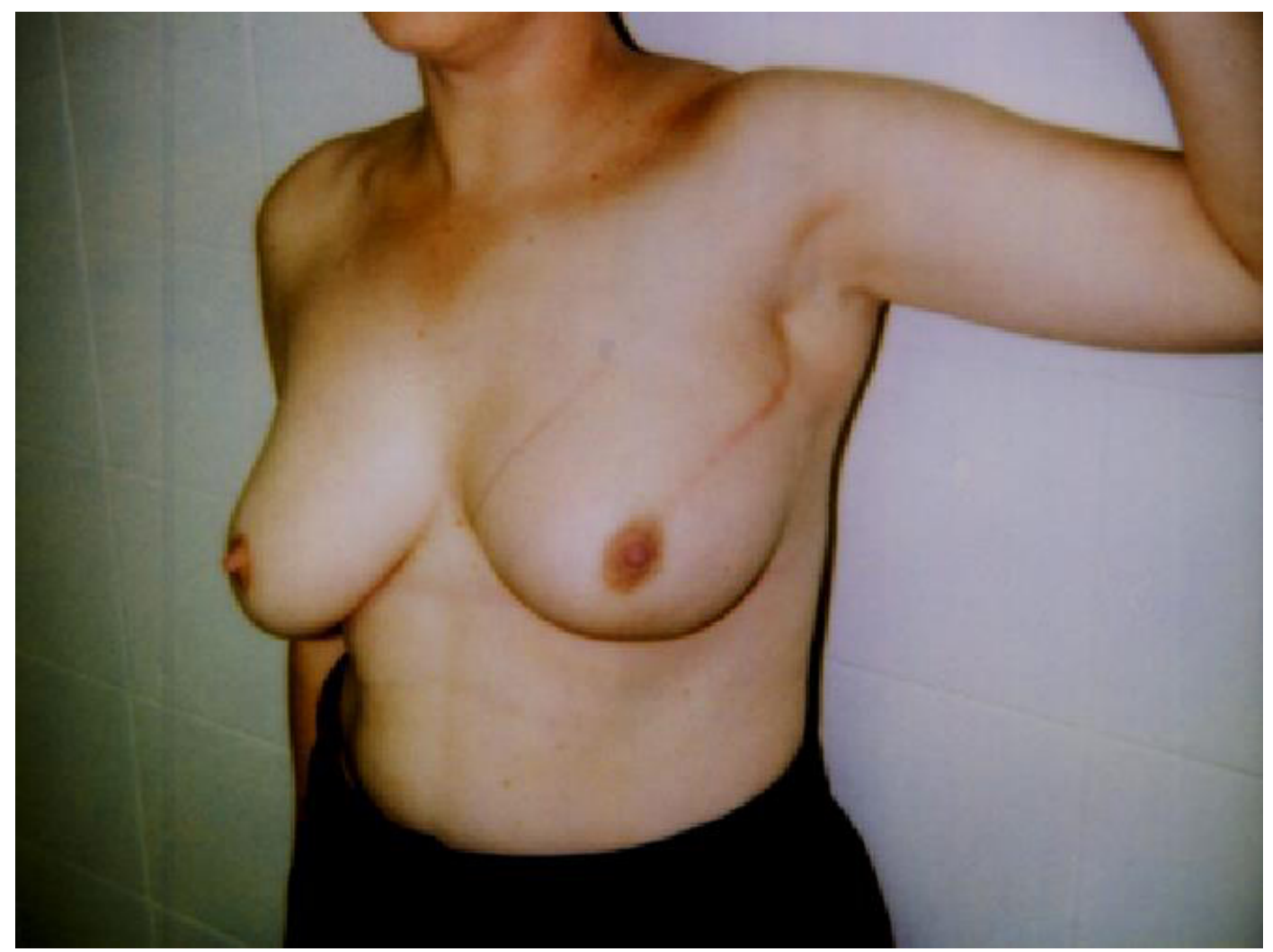

inicio, existe una tendencia actual a comenzar con quimioterapia y terapias antiHER2 (por ejemplo trastuzumab) antes de la cirugía, ya que ello permite valorar la eficacia de este tratamiento in vivo y permite predecir el pronóstico. Si la terapia neoadyuvante es capaz de hacer desaparecer el tumor en la pieza quirúrgica, las probabilidades de curación son muy altas. Tras la cirugía, la paciente debe recibir terapia antiHER2 (trastuzumab) hasta completar un año y radioterapia (también hormonas orales si el tumor además de HER2 expresa receptores hormonales).

3. En tumores demasiado grandes para poder realizar cirugía conservadora de la mama, debe comenzarse el tratamiento con quimioterapia y trastuzumab (denominada neoadyuvante). De nuevo, si se produce una desaparición completa del tumor, el pronóstico es excelente. La conservación de la mama es posible en un cierto porcentaje de los casos. Tras la cirugía, la paciente debe recibir terapia antiHER2 (trastuzumab) hasta completar un año y radioterapia (también hormonas orales si el tumor además de HER2 expresa receptores de estrógenos).

En los tumores triple negativos, el orden de terapias suele ser:

1. En tumores candidatos a cirugía conservadora de la mama de inicio (tumores pequeños con una relación tamaño de la mama/tamaño del tumor favorable): tumorectomía (cirugía conservadora de la mama) y biopsia del ganglio centinela. Tras ello, la paciente debe recibir quimioterapia y radioterapia.

2. En estos mismos tumores, aunque sean candidatos a cirugía conservadora de la mama de inicio, existe una tendencia actual a comenzar con quimioterapia antes de la cirugía, ya que estos tumores suelen ser quimiosensibles y además ello permite valorar la eficacia de este tratamiento in vivo y permite predecir el pronóstico. Si la terapia neoadyuvante es capaz de hacer desaparecer el tumor en la pieza quirúrgica, las probabilidades de curación son altas. Tras la cirugía, la paciente debe recibir radioterapia. 
En tumores demasiado grandes para poder realizar cirugía conservadora de la mama, debe comenzarse el tratamiento con quimioterapia (denominada neoadyuvante). De nuevo, si se produce una desaparición completa del tumor, el pronóstico es excelente. La conservación de la mama es posible en un cierto porcentaje de los casos.

\section{TRATAMIENTO DEL CÁNCER DE MAMA DISEMINA- DO (METASTÁSICO)}

Aproximadamente un $6 \%$ de las mujeres diagnosticadas de cáncer de mama en España presentan metástasis (extensión del cáncer a órganos distantes, como el pulmón o el hueso) en el momento del diagnóstico. Además, cerca del $20 \%$ de las enfermas operadas por tumores precoces sufren una recaída metastásica, pese a la progresiva eficacia de los tratamientos médicos actualmente disponibles.

Con contadas excepciones, el cáncer de mama diseminado es incurable, aunque existen tratamientos que pueden permitir controlar la enfermedad con buena calidad de vida durante años (López-Tarruella et al., 2015), en particular en los subtipos luminal y HER2.
La mayoría de las enfermas con tumores luminales metastásicos (que expresan receptores hormonales) pueden ser tratadas con varias líneas sucesivas de tratamiento hormonal, que suele ser capaz de controlar la enfermedad durante 3-4 años. Cuando el tratamiento hormonal ya no es eficaz, puede recurrirse a la quimioterapia. La mediana de supervivencia en cáncer de mama luminal metastásico se sitúa actualmente próxima a los 4 años. El subgrupo de cáncer de mama metastásico en que más avances se han producido en los últimos años es el de los tumores HER2-positivos. Se dispone de diversas terapias de diana anti-HER2 (trastuzumab, pertuzumab, T-DM1, lapatinib) que utilizadas consecutivamente (solas o en combinación con quimioterapia) permiten controlar la enfermedad durante mucho tiempo. La mediana de supervivencia en cáncer de mama HER2-positivo metastásico se sitúa actualmente próxima a los 5 años. Los tumores triple negativos tienen peor pronóstico, ya que la única opción disponible es la quimioterapia (aislada o en combinación con bevacizumab, un agente antiangiogénico).

\section{BIBLIOGRAFÍA}

Arroyo, A. (2003). Tendencias demográficas durante el siglo XX en España. En Arroyo, A. (coord.). Fecundidad. Madrid: Instituto Nacional de Estadística, pp. 119 - 162.

Bosch, X. (1998). Investigating the reasons for Spain's falling birth rate. Lancet, 352, 9131, p. 887.

Ferlay, J., Autier, P., Boniol, M., Heanue, M. Colombet, M. y Boyle, P. (2007). Estimates of the cancer incidence and mortality in Europe in 2006. Annals of Oncology, 18, 3, pp. 581-592. DOI: mdl498 [pii]10.1093/annonc/mdl498.

Fisher, B., Costantino, J. P., Wickerham, D. L., Redmond, C. K., Kavanah, M., Cronin, W. M., et al. (1998). Tamoxifen for prevention of breast cancer: report of the National Surgical Adjuvant Breast and Bowel Project P-1 Study. Journal of the National Cancer Institute, 90, 18, pp. 1371-1388.

Goss, P. E., Ingle, J. N., Ales-Martinez, J. E., Cheung, A. M., Chlebowski, R. T., Wactawski-Wende, J., et al. (2011). Exemestane for breast-cancer prevention in postmenopausal women. New England Journal of Medicine, 364, 25, pp. 2381 2391. DOI: 10.1056/NEJMoa1103507.
Hery, C., Ferlay, J., Boniol, M. y Autier, P. (2008). Quantification of changes in breast cancer incidence and mortality since 1990 in 35 countries with Caucasian-majority populations. Annals of Oncology, 19, 6, pp. 1187-1194. DOI: mdn025 [pii]10.1093/annonc/mdn025.

Levi, F., Te, V. C., Maspoli, M., Randimbison, L., Bulliard, J. L. y La Vecchia, C. (2007). Trends in breast cancer incidence among women under the age of forty. British Journal of Cancer, 97, 7, pp. 1013-1014. DOI: 6603919 [pii]10.1038/ sj.bjc.6603919.

López-Tarruella, S., Escudero, M., Martín, M., Jara, C., Guerreo, A., Lluch, A., Santaballa, A., Martínez del Prado, P., Lao, J., Alba, E., Fernández, A., Andrés, R., Llombart, A. et al. (2015). Impact on Survival of primary tumor resection in women with de novo metastatic breast cancer. The GEICAM Alamo I-III breast cancer registry (1990-2001) Cancer Research, 75, P2-13-17.

Martin, M., Llombart-Cussac, A., Lluch, A., Alba, E., Munarriz, B., Tusquets, I., et al. (2004). Estudio epidemiológico del grupo GEICAM sobre el cáncer de mama en España (1990-1993): proyecto «El Álamo» [Epidemiological study of the GEICAM group about breast cancer in Spain (1990-1993): «El Alamo» project]. Medicina Clínica, 122, 1, pp. 12-17. DOI: 13056182 [pii].

Perou, C. M., Sorlie, T., Eisen, M. B., van de Rijn, M., Jeffrey, S. S., Rees, C. A., et al. (2000). Molecular portraits of human breast tumours. Nature, 406, 6797, pp. 747-752. DOI: 10.1038/35021093.

Pollan, M., Pastor-Barriuso, R., Ardanaz, E., Arguelles, M., Martos, C., Galceran, J., et al. (2009). Recent changes in breast cancer incidence in Spain, 1980-2004. Journal of the National Cancer Institute, 101, 22, pp. 1584-1591. DOI: djp358 [pii]10.1093/jnci/djp358.

Soerjomataram, I., Pukkala, E., Brenner, H. y Coebergh, J. W. (2008). On the avoidability of breast cancer in industrialized societies: older mean age at first birth as an indicator of excess breast cancer risk. Breast Cancer Research and Treatment, 111, 2, pp. 297-302. DOI: 10.1007/s10549-007-9778-2. 